\title{
The Intraocular Changes of Anterior Segment Necrosis
}

\author{
ROSWELL R. PFISTER \\ Birmingham, Alabama
}

\begin{abstract}
Summary
Anterior segment necrosis is an acute or chronic process occasioned by embarrassment of the blood supply of the anterior segment of the eye. In the acute form this vascular obstruction leads to severe corneal oedema, necrosis of anterior uvea, hypotony and cataract formation. Depression of aqueous humour formation accounts for severe reduction of glucose levels in corneal stroma and aqueous humour lasting for two days after cautery of the long posterior ciliary arteries (LPCA) in rabbits. Lactate levels are initially significantly elevated but return to normal after one week. Stromal hydration was elevated for one week but then returned to normal. Corneal epithelial glycogen was diminished at one and two days after surgery but then returned to normal. Although unproven, oxygen deprivation probably plays a major role in endothelial ischaemia and therefore corneal oedema. It is concluded that the abnormalities seen in anterior segment necrosis stem from changes in aqueous metabolic components resulting from severely reduced aqueous turnover. Hyperbaric oxygen and intracameral metabolite substitution are unproven treatments but merit further experimental study.
\end{abstract}

Anterior segment necrosis (ASN) occurs as a chronic or acute process wherein the vascular supply of the anterior portion of the eye is compromised. Ischaemic ocular syndromes such as pulseless disease and the aortic arch syndrome represent a chronic ischaemia which is characterised by iris neovascularisation and atrophy, pupillary dilation and corneal oedema with uveitis. ${ }^{1,2}$ The acute form occasionally occurs after surgical procedures in which there is extensive disinsertion of the rectus muscles and/or obstruction of the long posterior ciliary arteries. ${ }^{3}$

In retinal detachment surgery, when three muscles are disinserted, especially the medial rectus, ASN is more likely to develop. These chances increase when the long posterior ciliary arteries are cauterised or when scleral buckling surgery is performed in patients manifesting haemoglobin SC. ${ }^{4,5}$ Strabismologists responding to a questionnaire reported a total of 30 cases of ASN for a calculated incidence of one in 1,333 strabismus operations. ${ }^{6}$ ASN is more common after the Hummelscheim transplantation operation in older individuals. . $^{7,9,10}$

Histopathological evidence of ASN has been verified in two studies. Iris and ciliary body necrosis were demonstrated in 21 of 150 eyes enucleated following transscleral diathermy, scleral resection, or polyethylene tubes for retinal detachment. ${ }^{11}$ A more recent study showed ASN present in $8.2 \%$ of 49 eyes obtained from 43 patients who previously underwent retinal detachment surgery. ${ }^{12}$

The clinical presentation of ASN is domi- 
nated by changes evident in the cornea and anterior uvea. Wrinkling of Descemets membrane and anterior uveitis with keratic precipitates herald the early picture of ASN. Later, massive corneal stromal oedema may be evident, followed by peripheral stromal infiltrates and hypotony.

\section{Anterior Segment Circulatory Considerations}

Anterior segment necrosis (ASN) is predicated on a failure of vascular perfusion of the anterior segment of the eye. Loss of the vascular supply to the ciliary body and iris results in partial necrosis of these tissues to a degree depending on the severity of the vascular deprivation. A decrease in the blood supply interferes with the active and passive transport of ions and nutritional substances into the aqueous humour by the non-pigmented epithelium of the ciliary body. This deprivation robs the cornea and crystalline lens of substances vital to their health.

In the human, the vascular supply of the anterior segment of the eye is dependent on two sources. The anterior ciliary arteries issue from the terminal arteries of the rectus muscles, perforating the sclera to join the greater arterial circle in the ciliary body at the root of the iris. The long posterior ciliary arteries branch (LPCA) from the ophthalmic artery deep in the orbit and progress anteriorly on the medial and lateral portions of the globe, intrasclerally. Additional branches given off by the LPCA in its intrascleral course penetrate sclera and travel in

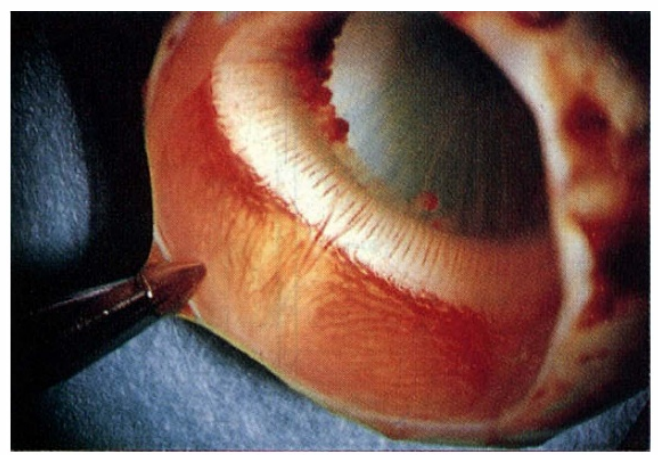

Fig 1 a. the suprachoroidal space to the ciliary body. The contribution each system makes to the anterior segment of the human eye is $40 \%$ anterior ciliary and $60 \%$ LPCA. In the rabbit the anterior ciliary system makes a very minor contribution to this system. ${ }^{13}$

Figure 1(a) shows the internal blood column in the normal rabbit eye and its interruption after LPCA cautery. (Fig 1(b)) In the rabbit eye transection of the four rectus muscles and perilimbal cautery has no effect on the corneal clarity. ${ }^{14}$ There is a slight fall in corneal epithelial glycogen but no effect on aqueous humour glucose, corneal hydration, or corneal aerobic glycolysis.

\section{Experimental Anterior Segment Necrosis}

Anterior segment necrosis has been induced in a number of animal models. Multiple disinsertions of the rectus muscles in monkeys ${ }^{8}$ or injury to the ciliary arteries around the optic nerve of rabbits has led to ASN.$^{15}$ Simple cutting of the long posterior ciliary arteries in rabbits caused no change in aqueous glucose, corneal transparency, or calcium uptake by the cornea. ${ }^{14,16}$ The most effective technique in rabbits was diathermy to both long posterior ciliary arteries resulting in extensive iris and ciliary body changes. ${ }^{17}$

In the experiments described here, a peritomy is performed in the anaesthetised rabbit and the medial and lateral rectus muscles are tenotomised. A radiodiathermy unit (MIRA) is used to place a continuous row of three or four transscleral cautery points along the course of the arteries from behind the equator

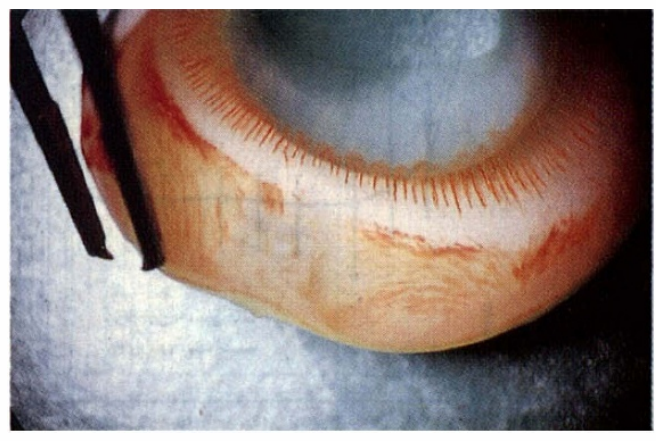

Fig 1 b.

Fig. 1. Interior view of long posterior ciliary artery $(a)$ in the normal and (b) after immediately after LPCA cautery. Note loss of blood column in $b$. 
to a position ahead of the rectus insertions. Conjunctiva is then pulled back to the limbus.

\section{General Anterior Segment Abnormalities}

The intraocular changes in experimentally produced ASN in rabbit eyes has been previously documented in our laboratory. ${ }^{18}$ The results noted here represent some citations from that study, subsequent new and unpublished data, and additional studies gleaned from the literature.

\section{Clinical Findings}

Anterior chamber

Cells and flare appeared in the aqueous humour immediately after cautery, reaching the maximum at seven days and disappearing after three weeks. The anterior chamber became shallow two to seven days later from posterior corneal swelling. At three to six weeks the cornea had returned to near normal thickness but cataract formation had swelled the lens sufficiently to maintain a narrow anterior chamber.

\section{Ciliary body and Iris}

Immediately after diathermy the pupil became dilated and vertically oval with collapse of the iris vasculature. After one day, the iris appeared pale, ultimately reducing to a velamentous frill with minimal circulation after three weeks. Mild hyphaema was occasionally present. Anderson has demonstrated rubeosis iridis beginning as a pink

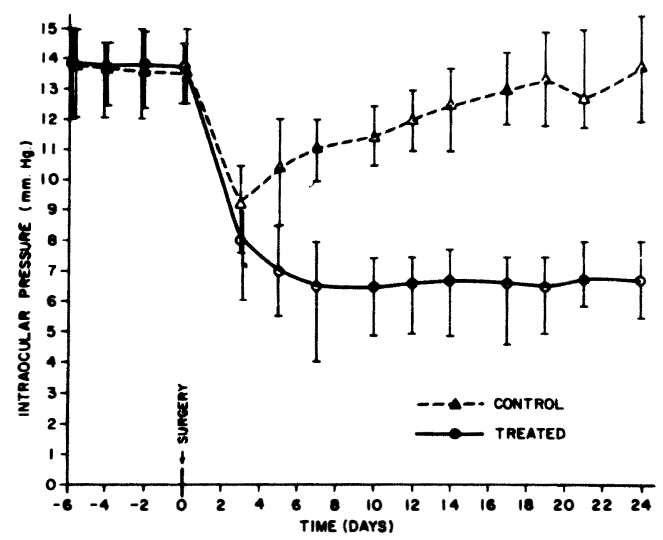

Fig. 2. Effect of cautery of both LPCA on the intraocular pressure in the squirrel monkey. (with permission from Levy et al, Invest Ophthalmol Vis Sci 1974, 13: 468-71.) blush at the junction of normal and atrophic iris, expanding extensively over the ensuing one to two weeks. ${ }^{19}$

\section{Lens}

Anterior subcapsular vacuoles appeared one day after diathermy. Water clefts between cortical lamellar fibres developed by seven days. Lens opacities progressed to a mature cataract at six weeks.

\section{Intraocular Pressure}

After LPCA cautery in the rabbit, ocular hypotony of less than five $\mathrm{mm} \mathrm{Hg}$ persisted for six weeks, though the intraocular pressure was higher after six weeks than in the first week. These results were confirmed by Guerry who showed that after LPCA cautery rabbit eyes show hyptony for weeks, ${ }^{21}$ some giving rise to phthisis bulbi. ${ }^{22}$ The squirrel monkey, with a ciliary circulation similar to humans, showed a depression of the IOP to about $50 \%$ after five days and for the subsequent 24 days. ${ }^{23}$ (Fig. 2) Although the surgery diminishes the volume of blood delivered to the ciliary body, additional hypotensive effect might be gained by damage to the long posterior ciliary nerves coursing in the same sheath. Proof for this is secured from chemical sympathectomy experiments in which a $35 \%$ reduction in the IOP was noted. ${ }^{24}$ It is likely that both mechanisms are operative to reduce IOP in the experimental model.

\section{Chemical Findings}

Aqueous glucose-Aqueous glucose was

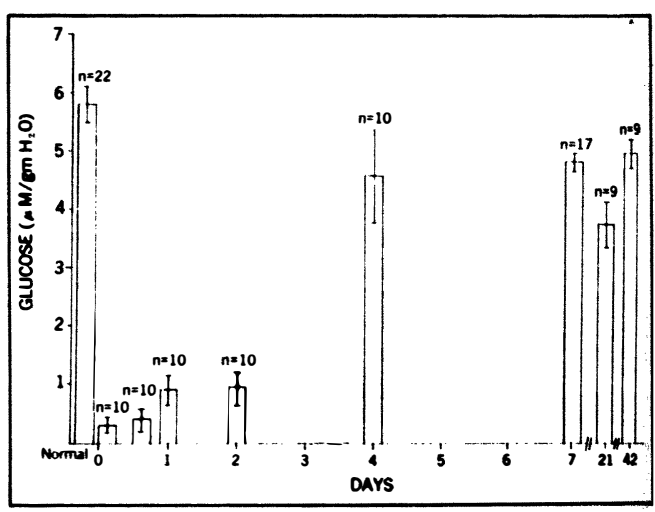

Fig. 3. Severe aqueous glucose depression after induced anterior segment necrosis lasted for two days but level was normal at 4,7 and 42 days (averages \pm $S E)$. 


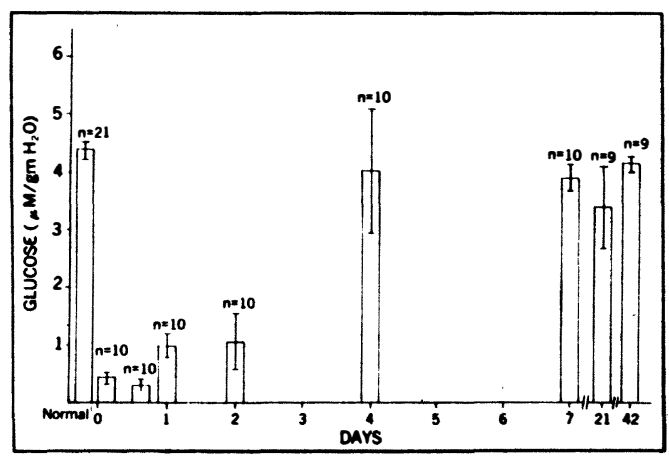

Fig. 4. Reduction of stromal glucose levels three hours after operation first returned to normal for four days and remained normal until 42 days (averages \pm $S E)$.

severely depressed from three hours to two days after diathermy of the LPCA $(\mathrm{P}<0.001$, Fig. 3). It remained in the low normal range between four days and six weeks except at three weeks when it was significantly below normal $(\mathrm{P}<0.001)$.

Stromal glucose-Stromal glucose showed a severe reduction from normal, three hours to two days after diathermy $(\mathrm{P}<0.001)$ (Fig. 4). It was normal thereafter.

Aqueous Lactate-There was a threefold increase in the lactate after three hours $(\mathrm{P}<0.001$, Fig. 5). This level then decreased for two days but remained higher than normal, $(\mathrm{P}<0.001)$ and was normal at four days. A further decrease to a subnormal level at one week $(\mathrm{P}<0.001)$ was followed by a return to normal at six weeks.

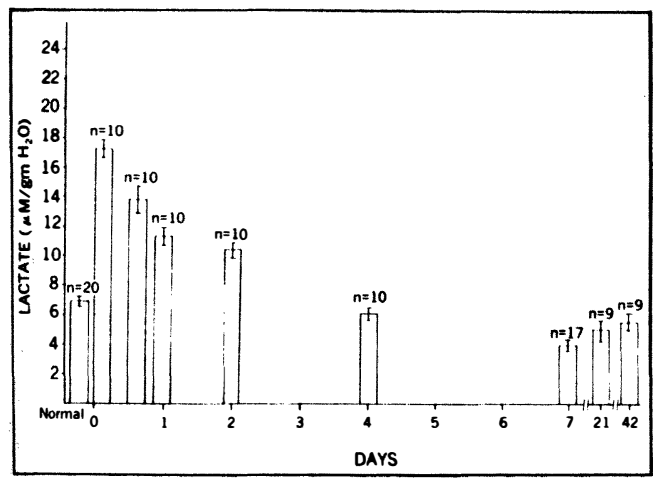

Fig. 5. Changes in aqueous humor lactate concentrations following long posterior ciliary cautery. Note decreasing levels of lactate falling to normal at four days and to its minimum at seven days. Return to normal was noted a 42 days (average $\pm S E$ ).
Stromal lactate-Figure 6 shows the large increase in lactate concentration after three hours $(\mathrm{P}<0.001)$ and normal lactate from 15 hours to four days. Thereafter, at seven, 21 and 42 days, lactate levels were below normal.

Electrolytes, $\mathrm{pH}$ and osmolarity-Preliminary analyses of these components of aqueous humour do not shed more light on the disturbances. The sodium, chloride and calcium levels were normal three hours, one day and seven days after operation. Aqueous potassium levels rose slightly from normal at three hours, but were $8.7 \mathrm{mg} \%$ at one day. Thereafter, aqueous potassium returned to normal. Aqueous osmolarity at 24 hours was 310 milliosmoles. Aqueous $\mathrm{pH}$ was normal from four days to six weeks.

Calcium transport-Surgical cutting of the LPCA in rabbits did not influence the transport of radioactive calcium into the cornea. ${ }^{16}$ It is difficult to compare these results to those obtained using cautery. The fact that the cornea remained clear postoperatively suggests that the obstruction to the artery was very temporary or incomplete.

\section{Corneal Effects of ASN}

The cornea is, for the most part, dependent on the aqueous humour for nutrition. The impact of LPCA cautery is to alter the composition and flow of the aqueous humour, thereby having a major effect on the cornea and the anterior segment of the eye.

Corneal hydration-After LPCA diathermy corneal oedema begins within hours.

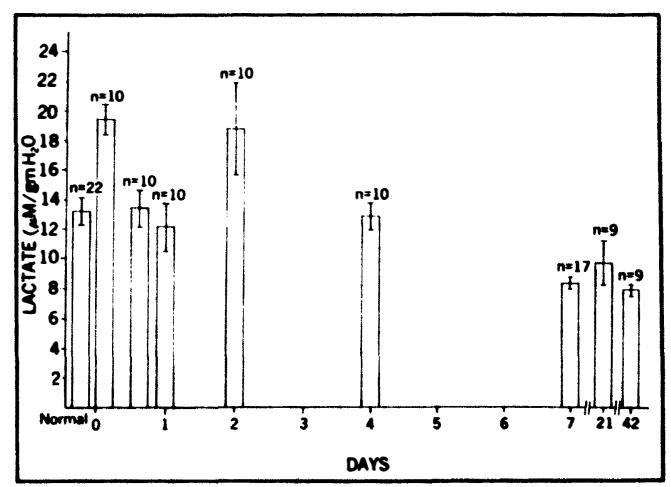

Fig. 6. Stromal lactate changes after operation. Note rapid elevation in three hours and normal values through four days. Lactate then dropped below normal (averages $\pm S E$ ). 


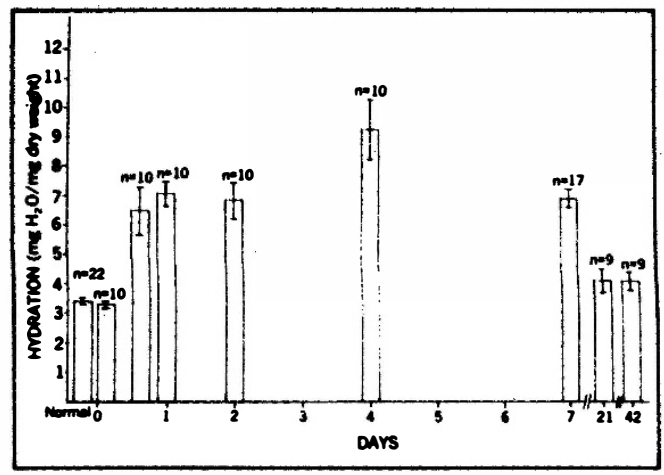

Fig. 7. Stromal hydration changes after operation. Hydration peaked at four days and was high normal at 21 days and 42 days (averages $\pm S E$ ).

Experimental hydration studies showed a very significant increase at 24 hours, reaching a maximum at four days (Fig. 7). Stromal hydration was still high at seven days but had subsided to high normal at 21 and 42 days.

Corneal histology-Mild hydropic changes were noted in the corneal epithelium but the haematoxyln and eosin stains were otherwise normal. Periodic acid Schiff stains showed moderate depletion of glycogen after 24 hours, and severe depletion, especially centrally, at two days. (Fig. 8) It was normal thereafter. Marked stromal oedema was present after four days, but this had disappeared by three weeks. Invasion of polymorphonuclear neutrophils (PMN) from the perilimbal arcades invaded the peripheral cornea at 15 hours, reaching a maximum between four to seven days. A sheet of polymorphonuclear

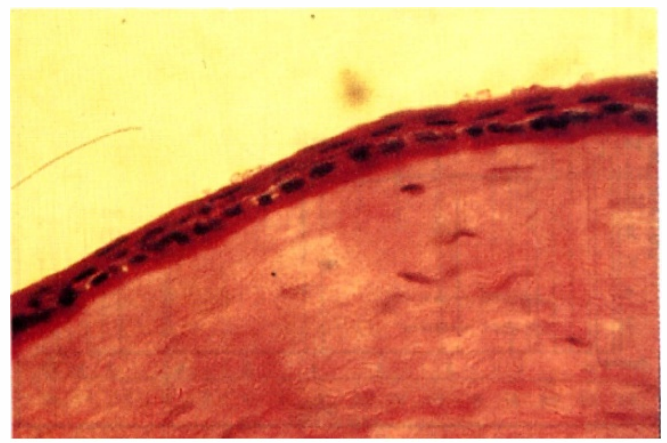

Fig 8a. leukocytes (PMN) appeared in predescements stroma during this period. At three and six weeks, monocytes had replaced the PMN.

The normal endothelium is characterised by a flat, polygonal array with numerous intracytoplasmic organelles. Within three hours after LPCA cautery there is hydropic . degeneration characterised by vacuoles within the cytoplasm and organelles, an irregular cellular profile, and retraction of some cell margins and tight junctions from adjacent cells (Fig. 9).

\section{Intraocular Effects on ASN}

Anterior chamber-Small numbers of PMN and red blood cells were noted up to seven days after LPCA cautery.

Ciliary body and Iris-Hydropic changes of the ciliary epithelium and iris at three hours were followed by the appearance of large patchy areas of ciliary body denuded of epithelium at 15 hours. Partial epithelial regrowth began between three to six weeks. The ciliary body stroma was oedematous up to three weeks, variously showing necrosis, cystoid spaces, and numerous widely dilated, empty blood vessels. At six weeks, iris and ciliary body were moderately atrophic and the vessels contained blood.

Lens-Six weeks after LPCA cautery all eyes showed mature, cataractous lenses, usually containing subcapsular calcium deposits. These results have been amplified by Fagerholm et al. who demonstrated lens fibre retraction, morgagnian spheres and membrane remnants in the subcapsular

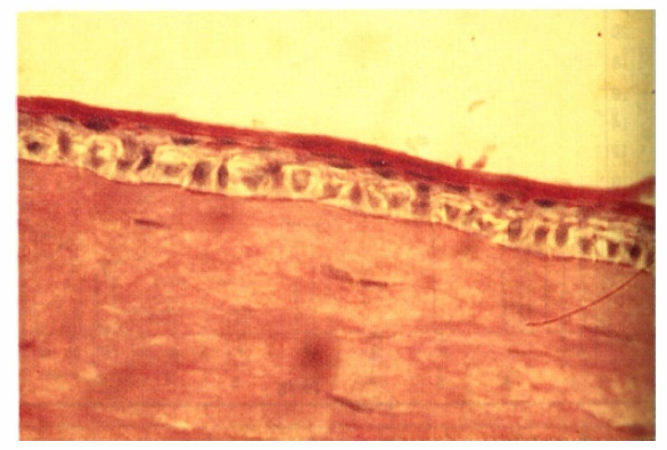

Fig 8 b.

Fig. 8. Normal epithelial glycogen was present (a) three hours after cautery but reduced significantly $(b)$ two days after LPCA cautery (PAS stain, ×200). 


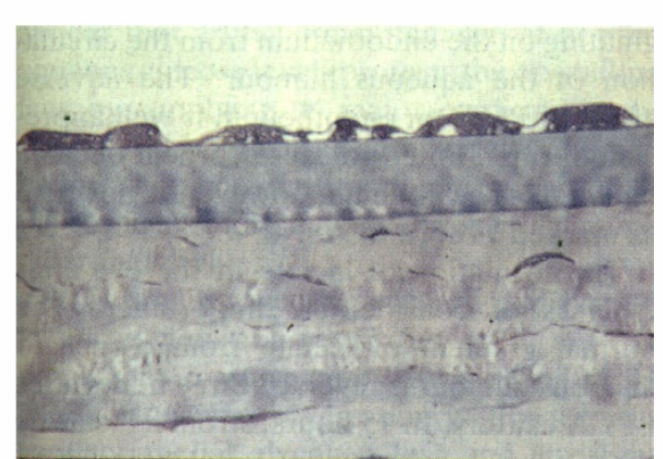

Fig. 9. Corneal endothelial cells are irregular, vacuolated and partially separated three hours after LPCA cautery.

zone. ${ }^{20}$ Six months after injury a new capsule formed which was continuous with, but posterior to, the original capsule. Elongated epitheloid cells, dispersed in a fibrous matrix, were found enclosed between the old and the new capsules. Amorphous electron-dense material or needle like crystals located in the cortex were identified by elemental analysis as calcium and phosphorus. The mechanism of deposition was thought to be mineral precipitation of hydroxyapatites on organic molecules.

\section{Discussion}

The body of data presented clearly indicate that interference with the circulation of the anterior segment of the eye predisposes to ASN. Animal models simulate the acute form of the disease; the primate model being most similar to the circulatory system in the anterior segment of the human eye. The immediate effect of this loss of blood supply is ocular hypotony. There is a breakdown in the blood-aqueous barrier occasioned by oxygen and metabolite deprivation. The outpouring of serum proteins and inflammatory cell ingress into all tissues adjacent to the anterior chamber testify to the enhanced vascular endothelial permeability. Curtailment of the blood supply of the anterior segment of the eye damages four vital intraocular structures: the iris, ciliary body, crystalline lens and cornea. The pupil dilates, probably from inadvertant injury to the long posterior ciliary nerves. The iris becomes atrophic and in the worse cases degenerates to a velamentous frill. The ciliary body desquamates large patches of non-pigmented and pigmented epithelium while the substance of the muscle undergoes cystic degeneration. Ultimately, the ciliary body atrophies with subsequent variable degrees of repair and regeneration. Hydration of the crystalline lens rapidly leads to a mature cataract. The cornea becomes oedematous, later developing peripheral infiltrates. The cornea will recover clarity in mild or moderate cases but remain oedematous, vascularise, and scar in the more severe forms. The cornea becomes nutritionally deprived, showing substantial swelling as a result. This swelling derives from impairment of endothelial cell function as a consequence of metabolite deprivation. The normal rabbit cornea relies on aqueous glucose for $90 \%$ of its substrate supply. Of the total substrate utilised $85 \%$ is converted to lactate and $15 \%$ is oxidised. ${ }^{25}$ In the absence of another substrate, Riley theorises that the cornea could convert lactate to pyruvate, oxidising it to carbon dioxide by the Krebs cycle. Furthermore, lactate and alpha ketoglutarate perfusion of the endothelium maintains normal corneal thickness. The presence of abundant mitochondria, enzymes of the Krebs cycle and corneal temperature reversal phenemena in the presence of lactate, all suggest at least a potential pathway for lactate metabolism. In spite of this, the large amount of lactate present in the aqueous humour might temporarily poison the endothelial cells.

Oxygen availability in the anterior segment after experimental ASN has not been formally studied. The classic concept of the oxygen profile across the normal cornea calculates that oxygen, at the level of the endothelium, is maintained at $50-55 \mathrm{~mm}$ $\mathrm{Hg} .{ }^{26}$ More recent data derived from an in vivo electrode slowly advancing through the cornea shows stromal oxygen levels falling from the anterior stroma to about $30 \mathrm{~mm} \mathrm{Hg}$ in the deep stroma. ${ }^{27} \mathrm{~A}$ sudden rise in the oxygen tension to $70 \mathrm{~mm} \mathrm{Hg}$ in the aqueous humour occurs once the endothelium is pierced and the anterior chamber entered. (Fig. 10) These data show that the endothelium is normally bathed by aqueous rich in oxygen and suggests that endothelium might require more oxygen for normal metabolism 


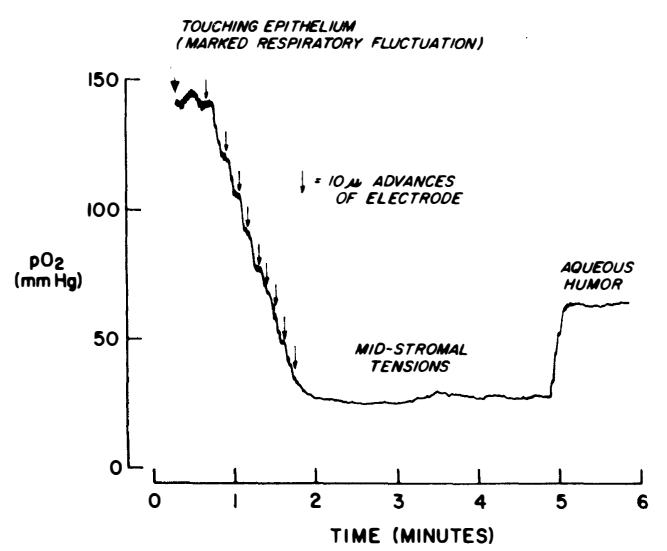

Fig. 10. Typical recording as the oxygen needle electrode traverses the cornea and enters the aqueous humour. (with permission from Kwan M, Niinikoski J and Hunt TK: Invest Ophthalmol Vis Sci 1972, 11: 10814.)

than can be provided by the diffusion of atmospheric oxygen. If this is true then aqueous humour oxygen (and therefore endothelial oxygen level) is likely to be substantially reduced in ASN to that in the stroma $(30 \mathrm{~mm}$ $\mathrm{Hg}$ ). The high level of aerobic activity in the endothelium would be curtailed, leading to severe corneal oedema. Takahashi supports this point of view with respect to the endothelium, by showing that an anoxic perfusate rapidly causes severe corneal oedema. ${ }^{28}$

On the supposition that oxygen deprivation was taking place in ASN, some workers have suggested that increasing the partial pressure of oxygen in the aqueous humour by hyperbaric oxygen might also be useful in therapeusis. Hyperbaric oxygen has been shown to increase the partial pressure of oxygen in the aqueous humour and preretinal vitreous in normal eyes. ${ }^{29}$ This treatment has already been used successfully in sickle cell haemoglobinopathy where sickling of red blood cells in the aqueous humour has been prevented by doubling transcorneal and intravascular oxygen. At this time it is unknown whether hyperbaric oxygen will be useful in the treatment of ASN.

Corneal oedema might also be caused, in part, by the presence of cells or substances inimical to the health of the endothelium. Evidence for this is the presence of PMN in a layer directly on the endothelium and mar- ginating on the endothelium from the circulation of the aqueous humour. The adverse effect of PMN on endothelium is well appreciated, often noted to cause corneal oedema in uveitis. The oedema, however, is nowhere as marked as seen in ASN.

The formation of lactate in the eye after ASN is of considerable importance. The normal gradient of lactate from cornea to aqueous humour is reversed three hours after LPCA cautery. In 15 hours, stromal lactate is again normal, but aqueous lactate is still high for three days. Excess lactate must not be coming from the cornea, but originating posteriorly, most likely from the ischaemic ciliary body. It is likely that a high level of anaerobic glycolysis proceeded in the locally hypoxia ciliary body processes and contained inflammatory exudate. A similar excess of lactate obtains anterior to intrastromal membranes. The presence of this metabolite did not protect the epithelium from delayed loss or subsequent ulceration. In ASN, glycogen stores in the epithelium were probably sufficient to tide the cornea over this interval of metabolite depletion.

The lens is entirely dependent on the aqueous humour for all of its nutritional supply. For this reason it is uniquely vulnerable to viscissitudes in the composition of the aqueous humour induced by reduction of the blood supply. The lens lacks tricarboxylic acid cycle enzymes, hence is unable to metabolise lactate. Significant reduction of the glucose supply and possible oxygen deprivation are therefore devastating to the lens epithelium and to the energetics necessary to maintain transparency. This explains the microscopic findings of swelling lens fibres and the ultimate deposition of calcium in dystrophic tissues. ${ }^{20}$ An insult to the lens of this magnitude can rarely reverse, resulting in the development of a mature cataract.

Preliminary unpublished data by Pfister has shown that after operation to induce ASN, continuous perfusion of the anterior chamber with BSS containing glucose, without sup. plementary oxygen, fails to keep the cornea from swelling severely. This infusion, via a fine, indwelling, intracameral silicone tube during the first four days after LPCA cautery; does keep the crystalline lens clear. It would 
appear that artificial maintenance of normal aqueous glucose levels protects the crystalline lens by supplying its major oxidisable substrate. The fact that the cornea is not affected by this supplemental treatment suggests that the cornea is more dependent on oxygen and other metabolites.

Experiments showing glucose deprivation in the aqueous humour after ASN are not proof that a cause and effect relationship exists. However, severe deprivation of glucose, epithelial glycogen loss and apparent inability to metabolite lactate augure strongly for a primary role of glucose, other metabolites, and oxygen in the pathophysiology of ASN.

Key words: anterior segment, aqueous humor, ciliary body, cornea, glucose, hydration, lactate, necrosis.

\section{References}

${ }^{1}$ Hedges TR: The Aortic arch syndromes. Arch Ophthalmol 1964, 71: 28-33.

${ }^{2}$ Knox DL: Ischemic ocular inflammation. Am J Ophthalmol 1965, 60: 995-1002.

${ }^{3}$ Boniuk $M$ and Zimmerman LE: Necrosis of the iris, ciliary body, lens and retina following scleral buckling operations with circling polyethylene tubes. 1961, 65: 671-93.

${ }^{4}$ Ryan SJ and Goldberg MF: Anterior segent ischemia following scleral buckling in sickle cell hemoglobinopathy. Am J Ophthalmol 1971, 72: 35-50.

${ }^{5}$ Eagle RC, Yanoff $M$ and Morse PH: Anterior segment necrosis following scleral buckling in hemoglobin SC disease. Am J Ophthalmol 1973, 426-33.

${ }^{6}$ France TD: Anterior segment ischemia syndrome following muscle surgery: the AAPO\&S experience. J Pediatr Ophthalmol Strabismus 1986, 23: 87-91.

${ }^{7}$ Stucchi C and Bianchi G: D'epigmentation en secteur de l'iris consecutive a des transplantation musculaires. Ophthalmol 1957, 133: 231-6.

${ }^{8}$ Girard LJ and Beltranena F: Early and late complications of extensive muscle surgery. Arch Ophthalmol 1960, 64: 576-84.

${ }^{9}$ Berens C and Girard LJ: Transplantation of the superior and inferior rectus muscles for paralysis of the lateral rectus. Am J Ophthalmol 1950, 33: 1041-9.

${ }^{10}$ Duguid IM: Anterior segment necrosis following retinal detachment surgery. Trans Ophthalmol Soc UK 1967, 87: 171-8.

${ }^{11}$ Boniuk $M$ and Zimmerman LE: Necrosis of uvea, sclera and retina following operations for retinal detachment. Arch Ophthalmol 1961, 66: 318-26.

${ }^{12}$ Wilson DJ and Green WR: Histopathologic study of the effect of retinal detachment surgery on 49 eyes obtained post mortem. Am J Ophthalmol 1987, 103: $167-79$.

${ }^{13}$ Prince JH: The Rabbit in Eye Research. Springfield Ill, Charles C Thomas, 1964, pp 522-3.

${ }^{14}$ Votockova J: The importance of the anterior ciliary arteries in the nutrition of the cornea. Cs Oftal 1962, 18: 1-7.

${ }^{15}$ Radnot $\mathbf{M}$ and Jobbagyi P: Vascularisation of the cornea in ischemia of the anterior segment. Klin Mbl Augenheilk 1968, 153: 840-4.

${ }^{16}$ Votockova J, Praus R, Sulcova H, Brettschneider I: The effect of blockade of the arteriaecililsres posteriores longae on the transparence and on the transport of $\mathrm{Ca}$ into the rabbit's cornea.

${ }^{17}$ Freeman MH, Hawkins WR, Schepens CL: Anterior Segment Necrosis. Arch Ophthalnol 1966, 75: 644-50.

${ }^{18}$ Pfister RR, Friend J, Dohlman CH: Anterior segment necrosis in rabbits. Arch Ophthalmol 1971, 86: 301-7.

${ }^{19}$ Anderson DM and Morin JD: Experimental anterior segment necrosis ans rubeosis iridis. Canad J Ophthalmol 1971, 6: 196-201.

${ }^{20}$ Fagerholm P, Lundevall, Trocme S, Wroblewski R: Human Experimental lens repair and calcification. Exp Eye Res 1986, 43: 965-72.

${ }^{21}$ Guerry D: Angiodiathermy of the long posterior ciliary arteries and its use in the treatment of glaucoma. Am J Ophthalmol 1944, 27: 1376-??.

${ }^{22}$ Wagenmann A: Experimentelle untersuchungen uber den einfluss der circulation in den netzhaut und aderhautgenfassen auf die ernahrung des auges, insbesondere der retina, und uber die folgen der sehnervendurchsneidung. Von Graefes Arch Ophthalmol 1980, 36: 1-??.

${ }^{23}$ Levy NS, Spector SS, Hood CI: Intraocular pressure following long posterior ciliary artery cautery in primates. Invest Ophthalmol 1974, 13: 468-71.

${ }^{24}$ Holland MG and Mims JL: Anterior segment chemical sympathectomy by 6-hydroxy-dopamine. I. Effect on intraocular pressure and facility of outflow. Invest Ophthalmol 1971, 10: 120-??.

${ }^{25}$ Riley M: Glucose and oxygen utilisation by the rabbit cornea. Exp Eye Res 1969, 8: 193-200.

${ }^{26}$ Fatt I: The steady-state distribution of oxygen and carbon dioxide in the in vivo cornea. Expl Eye Res 1968, 7: 103-12.

${ }^{27}$ Kwan M, Niinikoski, Hunt T: In vivo measurements of oxygen tension in the cornea, aqueous humor and anterior lens of the open eye. Invest Ophthalmol 1972, 11: 108-14.

${ }^{28}$ Takahashi GH: The relation of corneal anoxia to corneal hydration. Invest Ophthalmol 1967, 6: 562-??.

${ }^{29}$ Jampol LM: Oxygen therapy and intraocular oxygenation. Trans Am. Ophthalmol Soc 1987, 85: 407-37. 